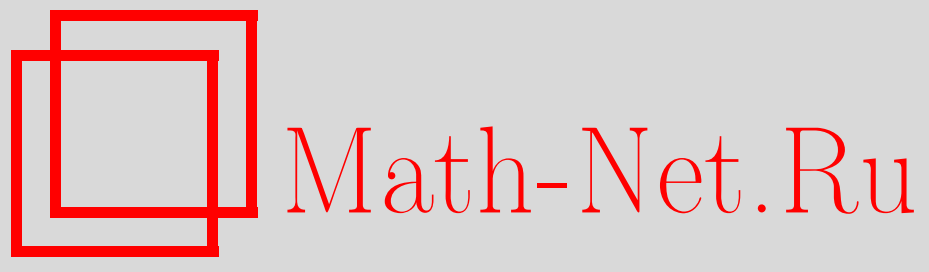

С. А. Терсенов, О некоторых постановках задач Коши и Дирихле, Матем. заметки, 2010, том 87, выпуск 1, 151155

DOI: https://doi.org/10.4213/mzm6620

Использование Общероссийского математического портала Math-Net.Ru подразумевает, что вы прочитали и согласны с пользовательским соглашением http://www . mathnet.ru/rus/agreement

Параметры загрузки:

IP: 54.197 .217 .227

26 апреля 2023 г., $17: 05: 05$

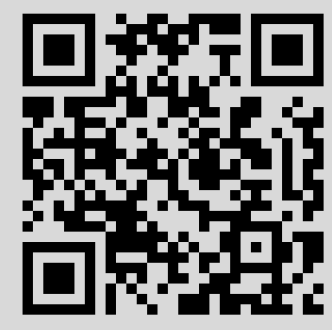




\section{О некоторых постановках задач Коши и Дирихле}

\section{С. А. Терсенов}

\section{1. Задача Коши.}

ЗАДАчА 1.1 . В области $Q=\mathbb{R}^{n} \times(t>0)$ рассмотрим параболическую систему уравнений

$$
\frac{\partial u}{\partial t}=\sum_{|k| \leqslant 2 b} A_{k}(x, t) D^{k} u, \quad u=\left(u_{1}, \ldots, u_{n}\right) .
$$

В области $t>0$ рассмотрим достаточно гладкие и ограниченные функции $t=f_{k}(x)$, $k=0, \ldots, n, \ldots, f_{0}(x)=0$, удовлетворяющие условию $\max _{\mathbb{R}^{n}} f_{k}(x)<\min _{\mathbb{R}^{n}} f_{k+1}, k=$ $0, \ldots, n, \ldots$ Р Рассмотрим задачу Коши в следующей постановке: найти в $Q$ ограниченное решение уравнения (1.1), удовлетворяющее условию

$$
u(x, 0)+\sum_{k=1}^{\infty} B_{k} u\left(x, f_{k}(x)\right)=f(x)=\left(f_{1}, \ldots, f_{n}\right),
$$

где $B_{k}-$ постоянные $n \times n$ матрицы.

Таким образом начальные условия даются как линейная комбинация значений решения на гиперплоскостях $t=f_{k}(x)$. Если $u(x, 0)=\varphi(x)$, то решение $u(x, t)$ дается формулой

$$
u(x, t)=\int_{\mathbb{R}^{n}} \Gamma(x, \xi, t) \varphi(\xi) d \xi,
$$

где $\Gamma(x, \xi, t)$ - фундаментальная матрица решений. Подставляя (1.3) в (1.2), получим систему интегральных уравнений

$$
\varphi(x)+\sum_{k=1}^{\infty} B_{k} \int_{\mathbb{R}^{n}} \Gamma\left(x, \xi, f_{k}(x)\right) \varphi(\xi) d \xi=f(x) .
$$

При помощи оценок (см. [1]) элементов матрицы $\Gamma(x, \xi, t)$ можно легко получить достаточные условия однозначной разрешимости системы уравнений (1.4) и задачи в терминах элементов матриц $B_{k}$.

Рассмотрим следующее уравнение параболического типа:

$$
\frac{\partial u}{\partial t}=L u \equiv \sum_{i, j=1}^{n} a_{i j} u_{x_{i} x_{j}}+\sum_{i=1}^{n} a_{i} u_{x_{i}}+a u, \quad a \leqslant 0 .
$$

В этом случае для $u(x, 0)=\varphi(x)$ будем иметь одно интегральное уравнение

$$
\varphi(x)+\sum_{k=1}^{\infty} d_{k} \int_{\mathbb{R}^{n}} \Gamma\left(x, \xi, f_{k}(x)\right) \varphi(\xi) d \xi=f(x),
$$

где $d_{k}$ - постоянные, а $\Gamma$ - фундаментальное решение (1.5).

Будем искать решение уравнения (1.6) методом последовательных приближений:

$$
\varphi_{0}(x)=f(x), \quad \varphi_{n}(x)=f(x)-\sum_{k=1}^{\infty} d_{k} \int_{\mathbb{R}^{n}} \Gamma\left(x, \xi, f_{k}(x)\right) \varphi_{n-1}(\xi) d \xi, \quad n=1,2, \ldots
$$


Имеем

$$
\varphi_{n+1}(x)-\varphi_{n}(x)=\sum_{k=1}^{\infty} d_{k} \int_{\mathbb{R}^{n}} \Gamma\left(x, \xi, f_{k}(x)\right)\left[\varphi_{n}(\xi)-\varphi_{n-1}(\xi)\right] d \xi, \quad n=0,1, \ldots
$$

Пусть $f(x) \in C\left(\mathbb{R}^{n}\right)$. Тогда в силу принципа максимума

$$
\left|\varphi_{n+1}(x)-\varphi_{n}(x)\right| \leqslant \delta^{n}|f|_{0}, \quad|f|_{0}=\max _{\mathbb{R}^{n}}|f|,
$$

где $\delta=\sum_{k=1}^{\infty}\left|d_{k}\right|$. Пусть $\delta<1$. Тогда $\varphi_{n}(x)$ сходится к непрерывной функции $\varphi(x)-$ решению уравнения (1.6), а решение задачи дается формулой типа (1.3).

Пусть $f(x) \in H^{2+\alpha}\left(\overline{\mathbb{R}}^{n}\right), a_{i j}, a_{i}$, а принадлежат $H^{2+\alpha, 1+\alpha / 2}(\overline{\mathbb{Q}})$, и пусть $f_{k}(x)=a_{k}-$ постоянные: $0=a_{0}<a_{1}<\cdots<a_{n}<\cdots$. Тогда $\varphi_{n}(x) \in H^{2+\alpha}\left(\overline{\mathbb{R}}^{n}\right)$ и при условии $c \delta<1$ последовательность $\varphi_{n}(x)$ сходится к $\varphi(x)$ в норме $H^{2+\alpha}\left(\overline{\mathbb{R}}^{n}\right)$ и $\varphi(x) \in H^{2+\alpha}\left(\overline{\mathbb{R}}^{n}\right)$, где $c>0$ не зависит от $f(x)$ (см. [2]).

При невыполнении условия $\delta<1$ соответствующая однородная задача в зависимости от $d_{k}$ и $a_{k}$ может иметь нетривиальное решение. Рассмотрим уравнение $u_{t}=u_{x x}$ и пусть при заданном $n$ имеем $1+\sum_{k=1}^{\infty} d_{k} e^{-n^{2} a_{k}}=0$. Тогда однородное уравнение, соответствующее (1.6), имеет нетривиальное решение $\varphi(x)=\cos n x$. Решением однородной задачи будет функция $u=e^{-n^{2} t} \cos n x$.

ЗАДАчА 1.2. Пусть снова $f_{k}=a_{k}$ - постоянные, $0=a_{0}<a_{1}<\cdots<a_{2 p}=T$. Рассмотрим в $Q$ полосы $Q_{s}=\mathbb{R}^{n} \times\left(a_{s}<t<a_{s+1}\right), s=0, \ldots, 2 p-1$. В полосе $Q_{s}$ рассмотрим параболическую систему уравнений вида

$$
(-1)^{s} u_{t}=L u,
$$

которая в $Q_{2 k}$ прямо параболическая, а в $Q_{2 k+1}$ обратно параболическая.

В области $Q_{T}=\sum_{s=0}^{2 p-1} Q_{s}$ рассмотрим задачу Коши в следующей постановке: найти непрерывную в $\bar{Q}_{T}$ функцию $u(x, t)$, удовлетворяющую условиям

1) $u(x, t)$ в полосах $Q_{s}$ является решением уравнения (1.9);

2) $u(x, t)$ удовлетворяет одному из начальных условий

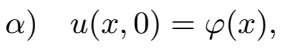

$$
\begin{aligned}
& \text { в) } u\left(x, a_{2 p}\right)=\psi(x) \text {. }
\end{aligned}
$$

Единственность. Обозначим через $u_{s}=u$ в $Q_{s}$. Пусть $\varphi=0$. Тогда $u_{0}=0$ в $Q_{0}$. В силу непрерывности решения в $\bar{Q}_{T}$ функция $u_{1}$ в $Q_{1}$ будет решением обратной задачи $-u_{1 t}=L u_{1}$ в $Q_{1}, u_{1}\left(x, a_{1}\right)=u_{0}\left(x, a_{1}\right)=0$. В силу единственности решения обратной задачи $u_{1}=0$. Продолжая этот процесс получим, что $u=0$ в $Q_{T}$. Аналогично единственность доказывается и в случае $\psi=0$.

Существование. Пусть постоянные $a_{s}$ удовлетворяют условию

$$
a_{2 s+2}-a_{2 s+1}=a_{2 s+1}-a_{2 s}, \quad s=0, \ldots, p-1,
$$

и пусть коэффициенты $L u$ не зависят от $t$. Тогда фундаментальное решение для прямо параболического уравнения будет иметь вид $\Gamma(x, \xi, t-\tau)$, а для обратно параболического $-\Gamma(x, \xi, \tau-t)$. Нетрудно видеть, что

$$
\begin{array}{rlrl}
u_{2 s} & =\int_{\mathbb{R}^{n}} \Gamma\left(x, \xi, t-a_{2 s}\right) \varphi(\xi) d \xi & \text { в } \quad Q_{2 s}, \\
u_{2 s+1} & =\int_{\mathbb{R}^{n}} \Gamma\left(x, \xi, a_{2 s+2}-t\right) \varphi(\xi) d \xi \quad \text { в } \quad Q_{2 s+1}
\end{array}
$$

и в силу (1.10) имеем $u_{2 s}\left(x, a_{2 s+2}-a_{2 s}\right)=u_{2 s+1}\left(x, a_{2 s+2}-a_{2 s+1}\right)$, что доказывает непрерывность $u(x, t)$ в $\bar{Q}_{T}$. 
ЗАДАчА 1.3 . В области $Q=\mathbb{R}^{n} \times(t>0)$ рассмотрим уравнение гиперболического типа

$$
u_{t t}=L u, \quad t>0,
$$

и задачу Коши в следующей постановке: найти решение $u(x, t)$ уравнения $(1.11)$, удовлетворяющее условиям

$$
u(x, 0)+\sum_{k=1}^{\infty} d_{k} u\left(x, f_{k}(x)\right)=\mu(x), \quad u_{t}(x, 0)+\sum_{k=1}^{\infty} d_{k} u_{t}\left(x, f_{k}(x)\right)=\nu(x) .
$$

Как и выше, имея представление решения обычной задачи Коши с начальными условиями $u(x, 0)=\varphi(x), u_{t}(x, 0)=\psi(x)$, условия (1.12) сведутся к исследованию системы уравнений относительно $\varphi(x)$ и $\psi(x)$. Рассмотрим случай уравнения колебания и пусть $f_{k}(x)=a_{k}$ :

$$
u_{t t}-u_{x x}=0, \quad t>0 .
$$

Представим решение уравнения (1.13) в виде

$$
u(x, t)=F(x-t)+\Phi(x+t), \quad F, \Phi \in C^{2}(\mathbb{R}) .
$$

Так как $u(x, 0)=F(x)+\Phi(x), u_{t}(x, 0)=-F^{\prime}(x)+\Phi^{\prime}(x)$, условия (1.12) относительно $F$ и $\Phi$ будут иметь вид

$$
\begin{array}{r}
F(x)+\Phi(x)+\sum_{k=1}^{\infty} d_{k}\left[F\left(x-a_{k}\right)+\Phi\left(x+a_{k}\right)\right]=\mu(x), \\
-F^{\prime}(x)+\Phi^{\prime}(x)+\sum_{k=1}^{\infty} d_{k}\left[-F^{\prime}\left(x-a_{k}\right)+\Phi^{\prime}\left(x+a_{k}\right)\right]=\nu(x) .
\end{array}
$$

Интегрируя (1.15), получим

$$
-F(x)+\Phi(x)+\sum_{k=1}^{\infty} d_{k}\left[-F\left(x-a_{k}\right)+\Phi\left(x+a_{k}\right)\right]=\int_{0}^{x} \nu(\xi) d \xi+C .
$$

Из (1.14) и (1.16) имеем

$$
F(x)+\sum_{k=1}^{\infty} d_{k} F\left(x-a_{k}\right)=\delta_{1}(x), \quad \Phi(x)+\sum_{k=1}^{\infty} d_{k} \Phi\left(x+a_{k}\right)=\delta_{2}(x),
$$

где

$$
\delta_{1}=\frac{1}{2} \mu(x)-\frac{1}{2} \int_{0}^{x} \nu(\xi) d \xi-\frac{1}{2} C, \quad \delta_{2}=\frac{1}{2} \mu(x)+\frac{1}{2} \int_{0}^{x} \nu(\xi) d \xi+\frac{1}{2} C .
$$

Пусть $\mu(x) \in C^{2}(\mathbb{R}), \nu(x) \in C^{1}(\mathbb{R})$. Для производных $F^{(s)}$ и $\Phi^{(s)}, s=0,1,2$, получим

$$
F^{(s)}+\sum_{k=1}^{\infty} d_{k} F^{(s)}\left(x-a_{k}\right)=\delta_{1}^{(s)}(x), \quad \Phi^{(s)}(x)+\sum_{k=1}^{\infty} d_{k} \Phi^{(s)}\left(x+a_{k}\right)=\delta_{2}^{(s)}(x) .
$$

Пусть выполняется условие $\delta<1$. Тогда применяя метод последовательных приближений, можно показать, что последовательные приближения $F_{n}^{(s)}(x), \Phi_{n}^{(s)}(x)$ сходятся равномерно к некоторым функциям $f_{s}(x), \varphi_{s}(x)$, причем $f_{0} \in C^{2}(\mathbb{R}), \varphi_{0} \in C^{2}(\mathbb{R}), f_{0}^{\prime}=f_{1}$, $f_{0}^{\prime \prime}=f_{2}, \varphi_{0}^{\prime}=\varphi_{1}, \varphi_{0}^{\prime \prime}=\varphi_{2}$.

Пусть $\mu=\nu=0$. Тогда решениями уравнений (1.17) будут

$$
F(x)=-\frac{1}{2} C \sum_{s=0}^{\infty}(-\beta)^{s}, \quad \Phi(x)=\frac{1}{2} C \sum_{s=0}^{\infty}(-\beta)^{s}, \quad \beta=\sum_{k=1}^{\infty} d_{k}<\infty .
$$


Отсюда $F(x)+\Phi(x)=0,-F^{\prime}(x)+\Phi^{\prime}(x)=0$, т.е. задача при условии $\mu=\nu=0$ и $\delta<1$ имеет решение и оно единственно. Если $\delta \geqslant 1$, то, как и выше, в зависимости от $d_{k}$ и $a_{k}$ однородная задача может иметь нетривиальное решение. Пусть $a_{k}=k \pi, d_{k}=(-1)^{k} \gamma_{k}$, $\sum_{k=1}^{\infty} \gamma_{k}=-1$, т.е. условие $\delta<1$ не выполняется. Решением однородной задачи будет функция $u(x, t)=a_{1} \sin (x-t)+a_{2} \cos (x+t), a_{1}, a_{2}$ - постоянные.

2. Задача Дирихле. В области $Q=\mathbb{R}^{n-1} \times\left(x_{n}>0\right)$ рассмотрим уравнение эллиптического типа порядка $2 n$

$$
L u=0,
$$

и пусть $x^{\prime}=\left(x_{1}, \ldots, x_{n-1}\right) \in \mathbb{R}^{n-1}$. Рассмотрим задачу Дирихле в $Q$ в следующей постановке: найти решение $(2.1)$ в $Q$, удовлетворяющее условиям

$$
\left.\frac{\partial^{s} u}{\partial x_{n}^{s}}\right|_{x_{n}=0}+\left.\sum_{k=1}^{\infty} d_{k s} \frac{\partial^{s} u}{\partial x_{n}^{s}}\right|_{x_{n}=f_{k}\left(x^{\prime}\right)}=f_{s}\left(x^{\prime}\right), \quad s=0, \ldots, n-1 .
$$

Как и выше, если известно интегральное представление обычной задачи Дирихле для $L u$ (см. [3]) при условиях

$$
\left.\frac{\partial^{s} u}{\partial x_{n}^{s}}\right|_{x_{n}=0}=\varphi_{s}\left(x^{\prime}\right), \quad s=0, \ldots, n-1,
$$

то подставив это представление для $\varphi_{s}\left(x^{\prime}\right)$ в $(2.2)$, получим систему интегральных уравнений. Ниже рассмотрим частные случаи этой задачи, когда $f_{k}=a_{k}$.

ЗАДАчА 2.1. В полупространстве $\mathbb{R}^{2} \times(z>0)$ рассмотрим уравнение Лапласа

$$
\Delta u \equiv u_{x x}+u_{y y}+u_{z z}=0 .
$$

Ищется гармоническая функция в $\mathbb{R}^{2} \times(z>0)$, удовлетворяющая условию

$$
u(x, y, 0)+\sum_{k=1}^{\infty} d_{k} u\left(x, y, a_{k}\right)=f(x, y),
$$

где $d_{k}$ - постоянные. Если $u(x, y, 0)=\varphi(x, y)$, то

$$
u(x, y, z)=\frac{z}{2 \pi} \int_{\mathbb{R}^{2}} \frac{f(\xi, \eta)}{\left[(x-\xi)^{2}+(y-\eta)^{2}+z^{2}\right]^{3 / 2}} d \xi d \eta
$$

и условие (2.3) принимает вид

$$
\varphi(x, y)+\sum_{k=1}^{\infty} d_{k} \frac{a_{k}}{2 \pi} \int_{\mathbb{R}^{2}} \frac{\varphi(\xi, \eta)}{\left[(x-\xi)^{2}+(y-\eta)^{2}+a_{k}^{2}\right]^{3 / 2}} d \xi d \eta=f(x, y) .
$$

В силу принципа максимума и при условии $\delta<1$, как и выше, нетрудно построить решение методом последовательных приближений. Более того, решение единственно.

ЗАДАчА 2.2. Рассмотрим теперь уравнение Лапласа

$$
\Delta u \equiv u_{x x}+u_{y y}=0
$$

в круге $x^{2}+y^{2}<1$. Пусть заданы числа $1>r_{1}>\cdots>r_{n}>\cdots$, где $r_{n}$ может стремиться к 0 . В круге $x^{2}+y^{2}<1$ ищется гармоническая функция $u(r, \varphi)$, удовлетворяющая условию

$$
u(1, \varphi)+\sum_{k=1}^{\infty} d_{k} u\left(r_{k}, \varphi\right)=f(\varphi)
$$


Представим гармоническую функцию $u(r, \varphi)$ в виде ряда

$$
u(r, \varphi)=\frac{1}{2} a_{0}+\sum_{s=1}^{\infty} r^{s}\left(a_{s} \cos s \varphi+b_{s} \sin s \varphi\right) .
$$

Подставляя (2.7) в (2.6), для определения $a_{s}, b_{s}$ получим следующую систему:

$$
a_{s} \varepsilon_{s}=\gamma_{s}, \quad s=0, \ldots, n, \ldots, \quad b_{s} \varepsilon_{s}=\beta_{s}, \quad s=1, \ldots, n, \ldots,
$$

где $\gamma_{s}$ и $\beta_{s}$ - коэффициенты Фурье функции $f(\varphi)$ и $\varepsilon_{s}=1+\sum_{k=1}^{\infty} d_{k} r_{k}^{s}$. Если $\varepsilon_{s} \neq 0$ для всех $s$, например это выполняется при условии $\delta<1$, то система (2.8) однозначно разрешима относительно $a_{s}, b_{s}$ и существует единственное решение задачи. Если $\varepsilon_{s}=0$, при $s=q_{1}, \ldots, q_{m}$, то задача разрешима только при условии, что коэффициенты Фурье $f(x)$, $\gamma_{s}=\beta_{s}=0$ для $s=q_{1}, \ldots, q_{m}$ и решение не единственно.

\section{СПИСОК ЦИТИРОВАННОЙ ЛИТЕРАТУРЫ}

[1] С. В. Эйдельман, Параболические системы, Наука, М., 1964. [2] О. А. Ладыженская, В. А. Солонников, Н. Н. Уральцева, Линейные и квазилинейные уравнения параболического типа, Наука, М., 1967. [3] И. Н. Векуа, Новые методы решения эллиптических уравнений, ОГИЗ, М.-Л., 1948.

\section{С. А. Терсенов}

Институт математики им. С. Л. Соболева СО РАН, Новосибирск
Поступило

26.09.2008

Исправленный вариант 16.08.2009 